Ensino, Saúde e Ambiente - V8 (1), pp. 15-25, A6ril, 2015

\title{
O ENSINO DA SAÚDE INDIGENA NOS CURRÍCULOS E ESPAÇOS ACADÊMICOS
}

\author{
THE TEACHING ABOUT INDIGENOUS HEALTH IN THE CURRICULA AND \\ OTHER ACADEMIC OPORTUNITIES
}

\author{
Nádile Juliane Costa de Castro \\ Universidade da Amazônia/Faculdade de Castanhal/ Núcleo de Altos Estudos Amazônicos-PPGDSTU- \\ UFPa/ nadiledecastro@hotmail.com
}

\begin{abstract}
RESUMO
O ensino sobre populações tradicionais vem ganhando espaço nos currículos e debates acadêmicos, sendo um tema em exponencial discussão nos cursos de formação em saúde, particularmente na Região Amazônica. O presente estudo baseou-se na vivência profissional da autora em Unidades Saúde da Família Indígena, localizadas no distrito sanitário indígena Guamá-Tocantins, estado do Pará (PA), Brasil e em quatro instituições de ensino superior, localizadas na região metropolitana de Belém-PA. Ao longo do processo do trabalho docente e ao analisar as grades curriculares das quatro instituições em que ocorreram as experiências, a autora constatou que apenas duas apresentaram atividades curriculares, cujo objetivo e ementa estavam direcionados de forma clara e específica às populações tradicionais ou grupos étnicos. Deste modo, foi percebida a necessidade dos componentes acadêmicos contemplarem as particularidades da população Amazônica em questão. Salienta-se ainda que a busca pelo conhecimento em populações tradicionais deve ocorrer ao longo do processo de formação do binômio discente/docente, por meio de pesquisa, redes de apoio da área, extensão e trocas de saberes.
\end{abstract}

Palavras-chave: Populações tradicionais; saúde indígena; currículo.

\begin{abstract}
The teaching about traditional populations is getting space in the curricula and academic debates, being a theme in exponential discussion on the courses of heath, particularly in the Amazon region. This study was based on the professional experience of the author in the Indigenous Family Health Units, located in indigenous health district Guamá-Tocantins, state of Pará (PA), Brazil and also in four institutions of higher education, located in the metropolitan area of Belém-PA. Along the teaching process, the author found that, when analyzing the curricula, only two out of four institutions had curricular activities, which were clearly and specifically directed to the traditional populations or ethnic groups. Thus, it was perceived the need for academic components contemplate these peculiarities of the Amazon population. It must be highlighted that the searching for knowledge in traditional populations should occur throughout the training process of the binomial student/ teacher, on the basis of research, support networks in the area, extension and knowledge exchanging.
\end{abstract}

Keywords: Traditional populations; Indigenous health, curriculum. 


\section{INTRODUÇÃO}

Nos últimos anos, a preocupação com a formação docente vem ganhando espaço na área educacional. Na década de 90, no quadro das mudanças sociais e tecnológicas que apresentaram novas maneiras de pensar, trabalhar e organizar o conhecimento, uma redefinição das práticas sociais tenderam a modificar os papéis sociais e profissionais, tradicionalmente atribuídos e constituídos. No campo da educação, a atuação dos professores vem sofrendo uma série de processos de mudanças e virtude da necessidade de readequação a esses novos paradigmas (NOVOA, 2000).

Segundo Tardiff (2000), os saberes dos professores trazem a marca das suas experiências pessoais, sendo, portanto, temporais, heterogêneas e situadas. Neste contexto de transformação, Vasconcelos (2005) cita que o papel do profissional, mais do que o de prestador de serviços junto a ações para a cura e a reabilitação, é a exigência da nova política de saúde, que exige um profissional adequado à consecução dos princípios do Sistema Único de Saúde (SUS) na prática diária.

Entre as mudanças observadas no cenário do ensino à saúde, identifica-se a necessidade de rediscutir os currículos, a fim de alcançar a excelência da formação acadêmica, implementando as habilidades do estudante para contemplar o contexto de grupos especiais, como é o caso das populações tradicionais, e em particular o conhecimento sobre as necessidades de saúde da população indígena.

Há experiências positivas com a inserção de disciplinas direcionadas a atuação junto à saúde das populações indígenas, pois se entende que na região norte, onde há a maior concentração de povos indígenas do Brasil (BRASIL, 2014 ), a realidade da diversidade da constituição étnica e cultural da população nativa canaliza a atenção à saúde a este grupo como prioritária e oportuniza a formação transcultural dos profissionais de saúde (SILVA, GONÇALVES e NETO, 2003).

Com base na problemática discutida, objetivou-se no presente estudo, realizar uma reflexão a respeito das atividades acadêmicas voltadas à saúde dos povos tradicionais indígenas por meio de experiência profissional, ações e estruturas acadêmicas vivenciadas e relatadas pela autora. 


\section{METODOLOGIA}

O presente estudo se baseou na vivência profissional-assistencial e profissionaldocente da autora em Unidades Saúde da Família Indígena localizadas no distrito sanitário indígena Guamá-Tocantins, estado do Pará e em quatro Instituições de Ensino Superior (IES), sendo estas, duas universidades e duas faculdades localizadas na região metropolitana de Belém do Pará.

O distrito Guamá-Tocantins possui um população de 7.724 indígenas e abrange 17 municípios do Pará, incluindo 74 aldeias e 24 etnias (BRASIL, 2014 ). As aldeias o qual foram vivenciadas as experiências profissionais-assistenciais, incluíram as etnias Amanayé e Anambé, constituídas de aproximadamente 300 indígenas. A vivência profissional-assistencial ocorreu em um período de oito meses, entre Julho de 2007 a março de 2008.

No contexto da experiência profissional-docente, foi analisado e vivenciado um total de sete atividades curriculares assistidas pela autora/docente nas quatro IES, assim descritas: Saúde Coletiva, Saúde Comunitária, Políticas de Saúde, Enfermagem e SUS, Semiologia, Saúde da criança e Saúde Indígena. A vivência profissional-docente ocorreu em um período de quatro anos, entre Agosto de 2010 a Novembro de 2014.

A apresentação dos resultados seguiu uma abordagem reflexivo-discussiva, com base no que foi vivenciado pela autora. Deste modo, este relato de experiência contextualizou a apresentação de disciplinas específicas sobre populações tradicionais e outras atividades curriculares afins, além da inserção do contexto amazônico nas matrizes curriculares.

\section{RESULTADOS E DISCUSSÃO}

\section{A Realidade do ensino em saúde sobre populações tradicionais}

A progressiva inserção das ações de saúde do SUS preconizadas pelo Ministério da Saúde (MS) nos povos tradicionais propicia que determinadas populações, como os indígenas sejam citados nos espaços acadêmicos, gerando assim, uma necessidade de discussão sobre a reformulação das atuais matrizes curriculares das IES, especialmente daquelas que possuem estreita relação com estas populações. Nesse aspecto, podem-se citar o caso das IES que pertencem a regiões contíguas a instalação de distritos 
indígenas, como é o caso da Universidade Federal do Amapá- campus binacional, localizado no município do Oiapoque- Amapá, uma das pioneiras no curso de Licenciatura Intercultural Indígena, no epicentro da etnia Galibi/Wajãpi (UNIFAP, 2014).

Apesar deste visível estreitamento entre a comunidade indígena e o ensino ser realidade em alguns estados da Amazônia brasileira, o ensino superior e os cursos da área de saúde ainda não possuem uma efetiva discussão das estratégias de saúde para as populações tradicionais, como os indígenas, ribeirinhos, e remanescentes quilombolas. Ainda é um espaço de discussão tênue, onde este tema ainda é muito restrito a disciplinas voltadas às ciências humanas, como a antropologia e a sociologia.

A concretização das mudanças poderão ser realizadas com diálogos mais efetivos por meio de oficinas, simpósios, e encontros acadêmicos, que permitam o debate e análise deste contexto, instigando no discente a percepção sobre a necessidade de discussão sobre a saúde indígena.

Segundo Ceccim e Ferla (2008):

A Educação Superior, como gestão setorial do ensino, teve pouca ocupação com a produção política que ocorreu no interior da área setorial da saúde. Foram as associações de ensino das profissões de saúde e o próprio Sistema Único de Saúde que se ocuparam do debate sobre a mudança na formação e a formulação de diretrizes curriculares.

O ensino superior por meio dos cursos de graduação e pós-graduação deve gerir juntamente com os professores e preceptores esta reformulação, pois consequentemente irá beneficiar a formação de profissionais de saúde da Amazônia. As instituições de ensino superior tem por meio do ensino a ferramenta para emergir novas perspectivas para a pesquisa e extensão na área dos estudos das populações indígenas. Experiências na pós-graduação latu sensu já estão sendo disponibilizadas pela Universidade Federal de São Paulo (UNIFESP) em parceria com o programa "Mais Médicos", do Governo Federal, a fim de melhorar e subsidiar a formação da categoria que trabalhará com essa população.

Devemos entender que essas mudanças permitem um novo olhar pelos profissionais docentes da área de saúde frente a necessidade de conduzir novas habilidades na formação acadêmicas, não apenas direcionando esta discussão entre disciplinas historicamente construídas a luz de temáticas étnicas e sociais, como também por meio de disciplinas de formação específica nos cursos de graduação. De 
acordo com Buchabqui, Cap e Petuco (2006), a própria noção de que nossa formação deve ser toda em sala de aula precisa ser revista e repensada.

\section{A deficiência do ensino em saúde sobre populações tradicionais nas IES amazônicas: Como contornar?}

Ao longo do processo do trabalho docente, a autora percebeu que, entre as grades curriculares das instituições onde ocorreram as experiências, apenas duas apresentaram disciplinas curriculares cujos objetivos e ementas estavam direcionados às populações tradicionais ou grupos étnicos de forma especifica, sendo uma IES da rede estadual e outra da rede privada. Estas IES abordam a temática por meio das disciplinas Assistência de enfermagem a populações tradicionais e Assistência de enfermagem a saúde indígena, respectivamente. As demais instituições não possuíam disciplinas específicas para a área. Entretanto, devemos considerar que todas as instituições durante a formulação do plano de ensino semestral, exigiram um tópico ou unidade que contemplasse o conteúdo por meio das habilidades pretendidas pela atividade disciplinar.

Os direcionamentos específicos em outras disciplinas não afins da temática em questão, se restringiram à exposições pontuais e com poucas oportunidades de diálogo e discussão, não permitindo um amplo debate a cerca do tema. Nos locais onde há disciplinas específicas ao tema, a autora conseguiu discutir o cenário indígena em vários aspectos e grupos etários, assim como morbidades mais frequentes nessas populações. Com a propiciação de uma carga horária mínima, é possível oportunizar a realização de atividades com abordagem interdisciplinar, permitindo assim, melhor compreensão dos aspectos biopsicossociais direcionados especialmente à saúde coletiva.

Silva, Gonçalves e Neto (2003), afirmam que:

Para a atuação em saúde indígena é essencial a compreensão do processo saúde-doença de forma ampliada, incluindo o aspecto étnico-cultural, e que o profissional busque se atualizar e adquirir novos conhecimentos. O profissional precisa estar preparado para atuar na atenção básica à saúde indígena, identificar fatores de risco e atuar preventivamente, planejar e implementar, em conjunto com a equipe as ações e programas, realizar acompanhamento, supervisão e avaliação do agente indígena de saúde e do auxiliar de enfermagem

Silva (2014) reitera que a inclusão da diversidade cultural no currículo é antes de tudo reconhecer as especificidades inerentes a determinados grupos sociais. É direcionar 
um olhar diferente do que ocorria no currículo anterior, sejam nos aspectos relacionados a gênero, crenças, etnias, etc. Compreender que não se trata apenas de trabalhar "a tolerância" em sala de aula ou realizar "breves visitas" à cultura do outro, ou ainda "contemplar" minorias que estavam excluídas ou mal representadas neste currículo.

No estudo de Marinelle et al. (2012), identificou-se que a ausência desse preparo proporciona ao profissional um sentimento de insegurança referente à assistência que deverá prestar, e que há necessidade de realização de capacitação prévia, e educação constante ao enfermeiro que destinará seu cuidado ao indígena, sendo esses fatores relevantes, no que diz respeito à qualificação da assistência, para que ele seja, então, competente, em relação a aspectos culturais, embasados em conhecimentos antropológicos. Por outro lado, o estreitamento das IES com comunidades indígenas pode propiciar ainda, a vivência da realidade por parte do binômio discente/docente, considerando a prática profissional de qualquer aspecto, indispensável para a formação continuada (MOREIRA, ANJOS e RÔÇAS, 2014).

Dessa forma entendemos a importância de rediscutir os currículos universitários a fim de desenvolver as habilidades profissionais por meio das disciplinas curriculares, objetivando formar técnicos em saúde conscientes desta possibilidade profissional, assim como também permiti desenvolver no professor a consciência em buscar conhecimento atual e práticas de ensino eficientes, seja por meio de suas vivências ou pela pesquisa pedagógica.

De acordo com o estudo de Oliveira (2013), os sujeitos da sua pesquisa optaram pela atuação na Saúde Indígena como primeiro emprego após a formação acadêmica e o fizeram, principalmente por oportunidade de trabalho. Estes enfermeiros têm, em média, mais experiência profissional calculada em anos dentro da Saúde Indígena do que aqueles provenientes de outras áreas da enfermagem. A outra metade dos enfermeiros migrou para a saúde indígena atraída pelo salário.

Em 2010 foi criada a Secretaria Especial de Saúde Indígena (Sesai) que pertence a área do Ministério da Saúde responsável por coordenar a Política Nacional de Atenção à Saúde dos Povos Indígenas e todo o processo de gestão do Subsistema de Atenção à Saúde Indígena (SasiSUS), no âmbito do Sistema Único de Saúde (SUS). Esta surgiu a partir da necessidade de reformulação da gestão da saúde indígena no país, demanda reivindicada pelos próprios indígenas durante as Conferências Nacionais de Saúde Indígena (BRASIL, 2014 $4^{\mathrm{b}}$ ). 
Rediscutimos aqui que a possibilidade empregatícia na área em questão é uma realidade implementada principalmente após a criação da Sesai, pois a autonomia da secretaria possibilitou uma discussão entre os profissionais já em prática clinica dentro das aldeias, gestores e representações indígenas. As práticas de saúde se fortaleceram amparadas conjuntamente com as diretrizes da Lei Orgânica da Saúde, norteando discussões de políticas de saúde inseridas para esta população, revendo condições de trabalho, reconhecendo a necessidade de considerar as diferenças culturais $\mathrm{e}$ fortalecendo as ações dentro da atenção básica que ocorre dentro das aldeias, que é fator essencial dentro das políticas de saúde aos povos indígenas.

\section{Contextualização da saúde indígena para desenvolvimento de habilidades específicas}

Para desenvolver as habilidades perante o assunto, foi necessário a busca ativa de artigos científicos, sites especializados, legislações e arquivos fotográficos e documentais da autora. É pertinente dizer que a autora atuou no período de 08 meses na assistência direta a saúde indígena na região do baixo Tocantins, o que permitiu ao longo do processo de formulação das aulas, a construção de conteúdo programático de acordo com as realidades encontradas, fazendo uma interface entre o preconizado pelo Ministério da Saúde (MS) e as realidades encontradas e observados durante a experiência profissional-assistencial.

Esta percepção da realidade estreitou a possibilidade de problematização do conteúdo, pois deste modo foi possível trazer para a sala de aula as peculiaridades culturais específicas de cada etnia, instigando tanto o professor como ao aluno a investigação sobre as interferências externas e/ou urbanas.

\footnotetext{
A educação está intimamente ligada à política cultural. O currículo nunca é apenas um conjunto neutro de conhecimentos, que de algum modo aparece nos textos e nas salas de aula de uma nação. Ele é sempre parte de uma tradição seletiva, resultado da seleção de alguém, da visão de algum grupo acerca do que seja conhecimento legítimo. É produto de tensões, conflitos e concessões culturais, políticas e econômicas que organizam e desorganizam um povo. (APPLE, 2009)
}

Segundo Tardiff (2000) o conhecimento do professor provêm de diversas fontes. Em seu trabalho, um professor se serve de sua cultura pessoal, que provém de sua história de vida e de sua cultura escolar anterior; ele também se apoia em certos conhecimentos disciplinares adquiridos na universidade, assim como em certos conhecimentos didáticos e pedagógicos oriundos de sua formação profissional; ele se 
apoia também naquilo que podemos chamar de conhecimentos curriculares veiculados pelos programas, guias e manuais escolares; ele se baseia em seu próprio saber ligado à experiência de trabalho, na experiência de certos professores e em tradições peculiares ao ofício de professor.

Podemos então considerar que o ser docente deve obter vivências profissionaisassistenciais para que haja uma interface do ensino prático, de modo a contextualizar a teoria com a prática. Em concordância disto, a autora conseguiu lecionar a disciplinas realizando estudo de casos observados durante a estadia nas aldeias citadas anteriormente, o que também despertou o interesse e curiosidade por parte dos discentes.

Para que o acadêmico compreendesse melhor o espaço geográfico e social das aldeias, foi exposto o material documental, incluindo fotos e registros. O material documental do período da vivência profissional-assistencial possibilitou a visualização ao discente além das entrelinhas dos periódicos e diálogos diretos. Durante a implementação do conteúdo programático foram realizadas atividades de pesquisa cuja habilidade envolveu pesquisa sobre alimentação, crenças, costumes, organização étnica, território, novas morbidades entre os povos tradicionais e a organização dos serviços de saúde. Estes assuntos foram abordados em forma de pesquisa, montagem de painel, banners, discussões em grupos, exposições e construção de tecnologias educativas direcionadas.

Para Braga (2014), o docente precisa pensar práticas que propiciem a formação, não só de enfermeiros, mas de cidadãos críticos e reflexivos, por meio da compreensão da realidade em que estão inseridos, e de forma que possam mudar as situaçõesproblema com que se deparam em seu cotidiano de trabalho.

No tocante a interdisciplinariedade, ao ministrar a disciplina de Semiologia da Enfermagem, considerada de conteúdo programático mais divergente em relação à saúde indígena, ainda sim possibilitou a realização da contextualização por meio do eixo "Teorias de Enfermagem" e explanação das realidades da região metropolitana de Belém, realizados na modalidade de atividade extraclasse expositiva para toda a comunidade acadêmica.

Ao longo dos quatro anos de experiência profissional-docente, a autora encontrou poucas referências sobre o tema, assim como materiais didáticos, ficando restrito a vídeos produzidos por autores independentes ou por meio de material produzido pela fundação Oswaldo Cruz (FIOCRUZ), como também artigos científicos 
que tratavam de semiotécnicas a grupos étnicos específicos, e alguns debates sobre a formação profissional. Esses materiais, entretanto, não discutiam de forma aprofundada o direcionamento ao ensino de saúde sobre os povos indígenas.

Essa dificuldade de encontrar material específico para a assistência à saúde que não envolvesse somente questões antropológicas evidencia a necessidade da criação de grupos de pesquisas que envolvam um amplo aspecto multiprofissional, não apenas direcionado a estudos sociais e antropológicos, mas que possibilitem abordagens do tema em outras esferas, desta forma permitindo um direcionamento dos conteúdos às necessidades profissionais.

E como contextualizar em sala de aula as características dos povos indígenas? A experiência profissional-assistencial na área permitiu a autora trazer para os espaços acadêmicos aqui discutidos, problemáticas e singularidades encontradas no contexto das aldeias Anambé e Amanayé, que incluíram a desnutrição, parasitoses, alcoolismo, doenças sexualmente transmissíveis e saber popular. Estas temáticas foram trabalhadas por meio de pesquisas direcionadas às bases culturais e a influencia na saúde destas populações.

\section{CONSIDERAÇÕES FINAIS}

A experimentação da autora na Saúde Indígena, área de atuação da disciplina ministrada, permitiu que a mesma fizesse uma problematização de forma mais clara e próxima da realidade. É necessário que além da experiência, haja por parte do profissional a sensibilidade de fazer a interferência de outros campos, de modo a permitir que ocorra a interdisciplinariedade durante as exposições, diálogos e dinâmicas.

O contexto interdisciplinar deve comtemplar as necessidades da população da amazônica. A busca pelo aperfeiçoamento deve ocorrer ao longo do processo de formação do docente, pelas suas pesquisas, redes de apoio da área, extensão e busca pelo conhecimento sobre o tema. Deve-se entender que a realidade de nossa região exige que os profissionais professores coloquem em prática as características amazônicas em cada conteúdo ministrado.

É necessário, além de seguir as exigências do Ministério da Educação (MEC), que o docente faça uma auto-avaliação a respeito do conteúdo ministrado, a fim de se permitir novos olhares e novas interferências, para que no final o discente tenha a 
capacidade de realizar as condutas necessárias aos usuários dos serviços de saúde de forma satisfatória.

Por fim, a autora entende que seja necessário que as novas grades curriculares possuam uma carga horária mínima direcionada ao estudo das populações tradicionais, para que atenda além das exigências do MEC a necessidade e demanda local.

\section{REFERÊNCIAS}

APPLE, M.W. A política do conhecimento oficial: faz sentido a idéia de um currículo nacional. In: MOREIRA, A. F. B.; SILVA, T.T. (Orgs.). Currículo, Cultura e Sociedade. São Paulo: Cortez, 11.ed. 2009, p. 59-91.

BRAGA, M. J.G. Ser professor: um estudo sobre a constituição identitária profissional do enfermeiro docente. Cadernos de Educação. v.25, n.25, p.98-117, 2014.

BRASIL- Portal Brasil ${ }^{a}$. Brasil tem quase 900 mil índios de 305 etnias e 274 idiomas. Acessado em 12/12/2014. Disponível em: http://www.brasil.gov.br/governo/2012/08/brasil-tem-quase-900-mil-indios-de-305etnias-e-274-idiomas

b. Conheça a secretaria- SESAI. Acessado em 01/12/2014. Disponível em: http://portalsaude.saude.gov.br/index.php/conheca-a-secretaria-sesai

BUCHABQUI, J. A.; CAPP, E.; PETUCO, D. R. S. Convivendo com agentes de transformação: a interdisciplinaridade no processo de ensino/aprendizado em saúde. Revista Brasileira de Educação Médica. v.30, n.1, p.32-38, 2006.

CECCIM, R. B.; FERLA, A. A. Educação e saúde: ensino e cidadania como travessia de fronteiras. Trabalho, educação e saúde. v.6, n.3, p. 443-456, 2008.

MARINELLI, N.P.; NASCIMENTO, D.F.; COSTA, A.I.P.; POSSO, M.B.S.; ARAÚJO, L.P.Assistência à população indígena: dificuldades encontradas por enfermeiros . Revista Univap, v.18, n.32, p.52-65, 2012.

MOREIRA, L.D.; ANJOS, M.B.; RÔÇAS, G. Prática de estágio supervisionado: Um caso de formação continuada de professores. Ensino, Saúde e Ambiente. v.7,n.1, p.1-9, 2014.

NÓVOA, A. Universidade e formação docente. Interface. v.4, n.7, p. 129-138, 2000.

OLIVEIRA, M.L.C. Análise da capacitação dos enfermeiros que atuam na atenção à saúde das populações indígenas. Dissertação (Mestrado em Enfermagem). Universidade Federal do Amazonas. 136f, 2013. 
SILVA, N. C.; GONÇALVES, M. J. F.; NETO, D. L. Enfermagem em saúde indígena: aplicando as diretrizes curriculares. Revista Brasileira de Enfermagem, v. 56, n. 4, p. 388-391, 2003.

SILVA, M. P. A temática indígena no currículo e a lei 11.645/2008: mais um desafio à realidade escolar. Revista Contexto. v.4, n.1-2, p. 111-126, 2014.

SILVA, N.C.; GONCALVES, M.J.F.; LOPES NETO, D. Enfermagem em saúde indígena: aplicando as diretrizes curriculares. Revista Brasileira de Enfermagem. v.56, n.4, p.388-391, 2003.

TARDIF, M. Saberes profissionais dos professores e conhecimentos universitários: elementos para uma epistemologia da prática profissional dos professores e suas conseqüências em relação à formação para o magistério. Revista Brasileira de Educação. n.13, p. 5-24, 2000.

UNIFAP- Universidade Federal do Amapá- campus binacional. Licenciatura Intercultural Indígena. Acessado em 10/12/2014. Disponível em: http://www2.unifap.br/oiapoque/

VASCONCELOS, C.M. Paradoxos da mudança no SUS. Tese (Doutorado em Saúde Coletiva), Faculdade de Ciências Médicas. Universidade Estadual de Campinas, Campinas, São Paulo (SP), 2005. 